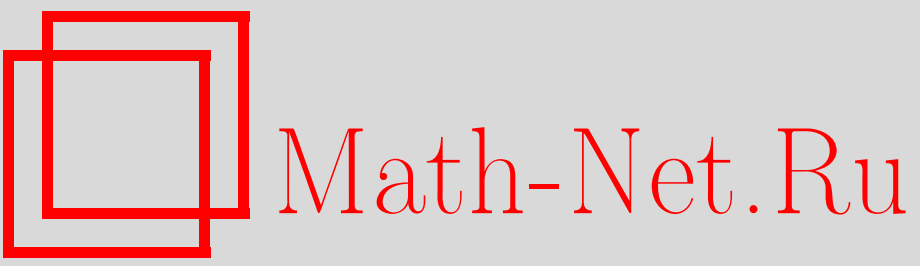

М. В. Плеханова, Сильное решение и задачи оптимального управления для класса линейных уравнений дробного порядка, Итоги науки и техн. Сер. Соврем. мат. и ее прил. Темат. обз., 2019, том 167, 42-51

DOI: https://doi.org/10.36535/0233-6723-2019-167-42-51

Использование Общероссийского математического портала Math-Net.Ru подразумевает, что вы прочитали и согласны с пользовательским соглашением

http://www.mathnet.ru/rus/agreement

Параметры загрузки:

IP: 52.90 .164 .192

26 апреля 2023 г., 02:24:01 


\title{
СИЛЬНОЕ РЕШЕНИЕ И ЗАДАЧИ ОПТИМАЛЬНОГО УПРАВЛЕНИЯ ДЛЯ КЛАССА ЛИНЕЙНЫХ УРАВНЕНИЙ ДРОБНОГО ПОРЯДКА
}

\author{
(c) 2019 г. $\quad$ М. В. ПЛЕХАНОВА
}

\begin{abstract}
АннотАция. Исследована однозначная разрешимость в смысле сильных решений задачи Коши для линейного неоднородного уравнения в банаховом пространстве, разрешенного относительно дробной производной Капуто. Предполагается, что оператор при искомой функции в правой части уравнения порождает аналитическое разрешающее семейство операторов для соответствующего однородного уравнения. Получен вид сильного решения исследуемой задачи Коши. Исследована разрешимость задач оптимального управления с выпуклым, полунепрерывным снизу, ограниченным снизу, коэрцитивным функционалом для рассмотренного уравнения. Общие результаты использованы для доказательства существования оптимального управления в задачах с конкретными функционалами. Абстрактные результаты для системы управления, описываемой уравнением в банаховом пространстве, проиллюстрированы на примерах задач оптимального управления для уравнения дробного порядка по времени, частными случаями которого являются уравнение субдиффузии и диффузионно-волновое уравнение.
\end{abstract}

Ключевъе слова: дробная производная Капуто, эволюционное уравнение дробного порядка, аналитическое в секторе разрешающее семейство операторов, задача оптимального управления, уравнение субдиффузии, диффузионно-волновое уравнение.

\section{STRONG SOLUTION AND OPTIMAL CONTROL PROBLEMS FOR A CLASS OF FRACTIONAL LINEAR EQUATIONS}

\section{(c) 2019 M. V. PLEKHANOVA}

Abstract. In this paper, we examine the unique solvability (in the sense of strong solutions) of the Cauchy problem for a linear inhomogeneous equation in a Banach space solved with respect to the Caputo fractional derivative. We assume that the operator acting on the unknown function in the right-hand side of the equation generates an analytic resolving operator family for the corresponding homogeneous equation. We obtain a representation of a strong solution of the Cauchy problem and examine the solvability of optimal control problems with a convex, lower semicontinuous, lower bounded, coercive functional for the equation considered. The general results obtained are used to prove the existence of an optimal control in problems with specific functionals. Abstract results obtained for a control system described by an equation in a Banach space are illustrated by examples of optimal control problems for a fractional equation whose special cases are the subdiffusion equation and the diffusion wave equation.

Keywords and phrases: Caputo fractional derivative, fractional evolution equation, resolving family of operators analytic in a sector, optimal control problem, subdiffusion equation, diffusionwave equation.

AMS Subject Classification: 49J20, 35R11, 34G10

Работа выполнена при поддержке Правительства РФ (постановление № 211 от 16.03.2013, соглашение № 02.А03.21.0011) и Министерства образования и науки РФ (гоударственное задание № 1.6462.2017/БЧ).. 
1. Введение. Рассматриваются вопросы разрешимости задачи оптимального управления

$$
\begin{gathered}
D_{t}^{\alpha} z(t)=A z(t)+B u(t)+f(t), \quad t \in(0, T), \\
z^{(k)}(0)=z_{k}, \quad k=0,1, \ldots, m-1, \\
u \in \mathcal{U}_{\partial}, \\
J(z, u) \rightarrow \inf ,
\end{gathered}
$$

где $D_{t}^{\alpha}$ - дробная производная Капуто, $m-1<\alpha \leqslant m \in \mathbb{N}$, замкнутый и плотно определенный в банаховом пространстве $\mathfrak{Z}$ оператор $A$ порождает аналитическое в секторе разрешающее семейство операторов соответствующего однородного уравнения, $f:(0, T) \rightarrow \mathfrak{Z}, \mathcal{U}$ - банахово пространство, $B \in \mathcal{L}(\mathcal{U} ; \mathfrak{Z}), \mathcal{U}_{\partial}$ - множество допустимых управлений, $J$ - функционал качества.

Вопросы существования и единственности решения задачи Коши (2) для однородного уравнения $D_{t}^{\alpha} z(t)=A z(t)$ изучались Э. Г. Бажлековой (см. [10]). Ею исследован класс операторов $\mathcal{A}^{\alpha}\left(\theta_{0}, a_{0}\right), \theta_{0} \in(\pi / 2, \pi), a_{0} \geqslant 0$, порождающих аналитическое в секторе разрешающее семейство операторов однородного уравнения; на основе результатов (см. [12]) найдены необходимые и достаточные условия принадлежности оператора этому классу. Отметим, что при $\alpha=1$ операторы такого класса называются секториальными и порождают аналитическую в секторе полугруппу операторов (см. [1-3,5]).

В настоящей работе получен новый результат о существовании единственного сильного решения задачи Коши $(1),(2)$ при $A \in \mathcal{A}^{\alpha}\left(\theta_{0}, a_{0}\right)$, представлен вид решения. Ранее в [11] аналогичный результат был получен в существенно более простом случае ограниченного оператора $A$; условия существования классического решения неоднородного уравнения с ограниченным оператором можно найти в [7].

Разрешимость задачи оптимального управления (1)-(4) исследована здесь с помощью теоремы А. В. Фурсикова (см. [9, теорема 2.3]). Полученный результат о задаче с абстрактным функционалом (4) использован для доказательства разрешимости задач оптимального управления с конкретными функционалами. Сформулированы условия, достаточные для единственности оптимального управления. Отметим, что разрешимость задачи управления для линейных эволюционных уравнений с вырожденным оператором при производной Капуто дробного порядка в случае относительной ограниченности пары операторов в уравнении была ранее исследована в [4].

Абстрактные результаты для системы управления, описываемой уравнением в банаховом пространстве, проиллюстрированы на примерах задач оптимального управления для уравнения дробного порядка по времени, частными случаями которого являются уравнение субдиффузии и диффузионно-волновое уравнение.

2. Сильное решение дробного уравнения с секториальным оператором. Пусть $\mathfrak{Z}-$ банахово пространство; обозначим через $\mathcal{L}(\mathfrak{Z})$ банахово пространство линейных непрерывных операторов из $\mathfrak{Z}$ в $\mathfrak{Z}$. Множество линейных замкнутых операторов с плотной в $\mathfrak{Z}$ областью определения, действующих в $\mathfrak{Z}$, будем обозначать $\mathcal{C l}(\mathfrak{Z})$.

Введем обозначения

$$
g_{\beta}(t)=\frac{t^{\beta-1}}{\Gamma(\beta)}, \quad J_{t}^{\beta} f(t)=\left(g_{\beta} * f\right)(t)=\int_{0}^{t} g_{\beta}(t-s) f(s) d s=\frac{1}{\Gamma(\beta)} \int_{0}^{t}(t-s)^{\beta-1} f(s) d s,
$$

где $\beta>0, t>0$. Пусть $m-1<\alpha \leqslant m \in \mathbb{N}, D_{t}^{m}$ - обычная производная целого порядка $m \in \mathbb{N}$. Производная Капуто задается равенством

$$
D_{t}^{\alpha} f(t)=D_{t}^{m} J_{t}^{m-\alpha}\left(f(t)-\sum_{k=0}^{m-1} f^{(k)}(0) g_{k+1}(t)\right) .
$$

Положим $\overline{\mathbb{R}}_{+}=\mathbb{R}_{+} \cup\{0\}$. Для оператора $A \in \mathcal{C l}(\mathfrak{Z})$ с областью определения $D_{A}$ при $\alpha>0$ рассмотрим уравнение

$$
D_{t}^{\alpha} z(t)=A z(t), \quad t \in \overline{\mathbb{R}}_{+} .
$$


Функция $z \in C\left(\overline{\mathbb{R}}_{+} ; D_{A}\right) \cap C^{m-1}\left(\overline{\mathbb{R}}_{+} ; \mathfrak{Z}\right)$, для которой

$$
g_{m-\alpha} *\left(z-\sum_{k=0}^{m-1} z^{(k)}(0) g_{k+1}\right) \in C^{m}\left(\overline{\mathbb{R}}_{+} ; \mathfrak{Z}\right),
$$

называется решением уравнения (5), если для всех $t \in \overline{\mathbb{R}}_{+}$выполняется равенство (5).

Следуя [10], множество операторов $\left\{Z(t) \in \mathcal{L}(\mathfrak{Z}): t \in \overline{\mathbb{R}}_{+}\right\}$будем называть семейством разрешающих операторов уравнения (5), если выполнены следующие условия:

(i) функция $Z(\cdot)$ сильно непрерывна на $\overline{\mathbb{R}}_{+}, Z(0)=I$;

(ii) для всех $t \in \overline{\mathbb{R}}_{+} Z(t)\left[D_{A}\right] \subset D_{A}$ при всяком $z_{0} \in D_{A}$ выполняется равенство $Z(t) A z_{0}=$ $A Z(t) z_{0}$

(iii) для любого $z_{0} \in D_{A}$ функция $Z(t) z_{0}$ является решением задачи Коши $z(0)=z_{0}, z^{(k)}(0)=0$, $k=1,2, \ldots, m-1$, для уравнения (5).

Следуя терминологии работы [10], будем говорить, что оператор $A \in \mathcal{C l}(\mathfrak{Z})$ принадлежит классу $\mathcal{A}^{\alpha}\left(\theta_{0}, a_{0}\right)$ для $\theta_{0} \in(\pi / 2, \pi), a_{0} \geqslant 0$, если существует семейство разрешающих операторов $\left\{Z(t) \in \mathcal{L}(\mathfrak{Z}): t \in \overline{\mathbb{R}}_{+}\right\}$для уравнения (5), допускающее аналитическое продолжение в сектор $\Sigma_{\theta_{0}} \equiv\left\{t \in \mathbb{C}:|\arg t|<\theta_{0}-\pi / 2, t \neq 0\right\}$, и для любых $\theta \in\left(\pi / 2, \theta_{0}\right), a>a_{0}$, существует такая константа $C(a, \theta)$, что для всех $t \in \Sigma_{\theta}$ выполняется неравенство

$$
\|Z(t)\|_{\mathcal{L}(\mathfrak{3})} \leqslant C(a, \theta) e^{a \operatorname{Re} t} .
$$

В соответствии с теоремой 2.14 из [10] (см. также более общую теорему I.2.1 в [12]) в случае $\alpha \in(0,2)$ оператор $A$ принадлежит классу $\mathcal{A}^{\alpha}\left(\theta_{0}, a_{0}\right)$ тогда и только тогда, когда выполнены следующие условия:

(i) для любого $\lambda \in S_{a_{0}, \theta_{0}} \equiv\left\{\mu \in \mathbb{C}:\left|\arg \left(\mu-a_{0}\right)\right|<\theta_{0}, \mu \neq a_{0}\right\}$ выполняется включение $\lambda^{\alpha} \in \rho(A) \equiv\left\{\mu \in \mathbb{C}: R_{\mu}(A) \in \mathcal{L}(\mathfrak{Z})\right\}$

(ii) для любых $a>a_{0}, \theta \in\left(\pi / 2, \theta_{0}\right)$ существует такая константа $K=K(a, \theta)>0$, что для каждого $\mu \in S_{a, \theta}$ выполняется неравенство

$$
\left\|R_{\mu^{\alpha}}(A)\right\|_{\mathcal{L}(\mathfrak{Z})} \leqslant \frac{K(a, \theta)}{\left|\mu^{\alpha-1}(\mu-a)\right|}
$$

здесь $R_{\mu}(A):=(\mu I-A)^{-1}$.

Лемма 1 (см. [6]). Пусть $\alpha>0, A \in \mathcal{A}^{\alpha}\left(\theta_{0}, a_{0}\right), \Gamma=\partial S_{a_{1}, \theta_{1}}$ при некоторых $a_{1}>a_{0}, \theta_{1} \in$ $\left(\pi / 2, \theta_{0}\right)$. Тогда семейство операторов

$$
\left\{Z_{\alpha, \beta}(t)=\frac{1}{2 \pi i} \int_{\Gamma} \mu^{\alpha-\beta} R_{\mu^{\alpha}}(A) e^{\mu t} d \mu \in \mathcal{L}(\mathfrak{Z}): t \in \mathbb{R}_{+}\right\}, \quad \beta \in \mathbb{R},
$$

допускает аналитическое продолжение в $\Sigma_{\theta_{0}} \equiv\left\{t \in \mathbb{C}:|\arg t|<\theta_{0}-\pi / 2, t \neq 0\right\}$. Для любых $a>a_{0}, \theta \in\left(\pi / 2, \theta_{0}\right)$ существует такое $C_{\beta}=C_{\beta}(a, \theta)$, что для каждого $t \in \Sigma_{\theta}$ выполняются неравенства

$$
\begin{array}{ll}
\left\|Z_{\alpha, \beta}(t)\right\|_{\mathcal{L}(\mathfrak{Z})} \leqslant C_{\beta}(a, \theta) e^{a \operatorname{Re} \tau}\left(|t|^{-1}+a\right)^{1-\beta}, & \beta \leqslant 1, \\
\left\|Z_{\alpha, \beta}(t)\right\|_{\mathcal{L}(\mathfrak{Z})} \leqslant C_{\beta}(a, \theta) e^{a \operatorname{Re} \tau}|t|^{\beta-1}, & \beta>1 .
\end{array}
$$

При этом $\lim _{t \rightarrow 0+} Z_{\alpha, \beta}(t)=0$ для $\beta>1$.

Рассмотрим задачу Коши

$$
z^{(k)}(0)=z_{k}, \quad k=0,1, \ldots, m-1,
$$

для неоднородного уравнения

$$
D_{t}^{\alpha} z(t)=A z(t)+f(t), \quad t \in(0, T),
$$


где $A \in \mathcal{A}^{\alpha}\left(\theta_{0}, a_{0}\right), f:(0, T) \rightarrow \mathfrak{Z}, T>0$. Пусть $q>1$. Сильным решением задачи (8), (9) называется функция $z \in L_{q}\left(0, T ; D_{A}\right) \cap C^{m-1}([0, T] ; \mathfrak{Z})$, для которой

$$
g_{m-\alpha} *\left(z-\sum_{k=0}^{m-1} z^{(k)}(0) g_{k+1}\right) \in W_{q}^{m}(0, T ; \mathfrak{Z}),
$$

выполнены равенства (8) и для почти всех $t \in(0, T)$ - равенство (9).

Теорема 1. Пусть $\alpha>0, \alpha-m+1>1 / q, A \in \mathcal{A}^{\alpha}\left(\theta_{0}, a_{0}\right), f \in L_{q}\left(0, T ; D_{A}\right)$. Тогда для любых $z_{k} \in D_{A}, k=0,1, \ldots, m-1$, существует единственное сильное решение задачи (8), (9), имеющее вид

$$
z(t)=\sum_{k=0}^{m-1} Z_{\alpha, k+1}(t) z_{k}+\int_{0}^{t} Z_{\alpha, \alpha}(t-s) f(s) d s
$$

и удовлетворяющее неравенству

$$
\|z\|_{C^{m-1}([0, T] ; 3)}+\left\|D_{t}^{\alpha} z\right\|_{L_{q}(0, T ; 3)} \leqslant C\left(\sum_{k=0}^{m-1}\left\|z_{k}\right\|_{D_{A}}+\|f\|_{L_{q}\left(0, T ; D_{A}\right)}\right)
$$

при некотором $C>0$, не зависящем от $z_{k}, k=0,1, \ldots, m-1, f$.

Доказательство. В силу результатов [10] функция

$$
\sum_{k=0}^{m-1} Z_{\alpha, k+1}(t) z_{k}
$$

является классическим решением, а значит, и сильным решением задачи (5), (8). Остается показать, что

$$
Z_{f}(t)=\int_{0}^{t} Z_{\alpha, \alpha}(t-s) f(s) d s
$$

является сильным решением задачи $z^{(k)}(0)=0, k=0,1, \ldots, m-1$, для уравнения (9).

Согласно (7) имеем

$$
\lim _{t \rightarrow 0+} Z_{\alpha, \alpha}^{(k)}(t)=\lim _{t \rightarrow 0+} Z_{\alpha, \alpha-k}(t)=0
$$

при $\alpha>1, k=0,1, \ldots, m-2$, поскольку в таком случае $\alpha-k>1$. Таким образом,

$$
Z_{f}^{(k)}(t)=\int_{0}^{t} Z_{\alpha, \alpha}^{(k)}(t-s) f(s) d s
$$

для $k=0,1, \ldots, m-1$.

В силу (6) при $f \in L_{q}\left(0, T ; D_{A}\right)$ согласно неравенству Гельдера имеем

$$
\begin{aligned}
\left\|Z_{f}^{(m-1)}(t)\right\|_{\mathfrak{Z}}=\left\|\int_{0}^{t} Z_{\alpha, \alpha-m+1}(t-s) f(s) d t\right\|_{\mathfrak{Z}} \leqslant C_{1} \int_{0}^{t}(t-s)^{\alpha-m}\|f(s)\|_{\mathfrak{Z}} d s \leqslant & \leqslant C_{2} t^{\alpha-m+1-\frac{1}{q}}\|f\|_{L_{q}\left(0, T ; D_{A}\right)} \rightarrow 0
\end{aligned}
$$

при $t \rightarrow 0+$, так как $\alpha-m+1>1 / q$.

Обозначим через $\mathfrak{L}[h]$ преобразование Лапласа функции $h$, а через $\mathfrak{L}^{-1}[H]$ - обратное преобразование для $H$. Доопределим $f$ нулем вне $(0, T)$; тогда $Z_{f}=Z_{\alpha, \alpha} * f$ является сверткой. В силу того, что

имеем

$$
\left\|\frac{R_{\mu^{\alpha}}(A)}{\lambda-\mu}\right\|_{\mathcal{L}(\mathfrak{Z})} \leqslant \frac{C}{|\mu|^{1+\alpha}}
$$




$$
\begin{aligned}
\mathfrak{L}\left[Z_{f}\right](\lambda)=\mathfrak{L}\left[Z_{\alpha, \alpha}\right](\lambda) \mathfrak{L}[f](\lambda)=\frac{1}{2 \pi i} \int_{0}^{\infty} e^{-\lambda t} \int_{\Gamma} R_{\mu^{\alpha}}(A) e^{\mu t} d \mu d t \mathfrak{L}[f](\lambda)= \\
\quad=\frac{1}{2 \pi i} \int_{\Gamma} \frac{R_{\mu^{\alpha}}(A)}{\lambda-\mu} d \mu \mathfrak{L}[f](\lambda)=R_{\lambda^{\alpha}}(A) \mathfrak{L}[f](\lambda) .
\end{aligned}
$$

По формуле для преобразования Лапласа от дробной производной с учетом замкнутости оператора $A$ и его коммутирования с собственной резольвентой получаем

$$
\begin{aligned}
\mathfrak{L}\left[D_{t}^{\alpha} Z_{f}\right](\lambda)= & \lambda^{\alpha} \mathfrak{L}\left[Z_{f}\right](\lambda)-\sum_{l=0}^{m-1} \lambda^{\alpha-l-1} Z_{f}^{(l)}(0)=\lambda^{\alpha} R_{\lambda^{\alpha}}(A) \mathfrak{L}[f](\lambda)= \\
& =R_{\lambda^{\alpha}}(A) A \mathfrak{L}[f](\lambda)+\mathfrak{L}[f](\lambda)=R_{\lambda^{\alpha}}(A) \mathfrak{L}[A f](\lambda)+\mathfrak{L}[f](\lambda)=\mathfrak{L}\left[Z_{A f}\right](\lambda)+\mathfrak{L}[f](\lambda),
\end{aligned}
$$

поэтому $D_{t}^{\alpha} Z_{f}=Z_{A f}+f=A Z_{f}+f$.

Покажем, что $D_{t}^{\alpha} Z_{f} \in L_{q}(0, T ; \mathfrak{Z})$. Так как $f \in L_{q}\left(0, T ; D_{A}\right)$, остается показать, что $A Z_{f} \in$ $L_{q}(0, T ; \mathfrak{Z})$. В силу оценок $(6),(7)$ при $\alpha \geqslant 1$ имеем

$$
\begin{aligned}
\left\|A Z_{f}\right\|_{L_{q}(0, T ; \mathfrak{Z})}^{q}= & \left\|\int_{0}^{t} Z_{\alpha, \alpha}(t-s) A f(s) d s\right\|_{L_{q}(0, T ; 3)}^{q}=\int_{0}^{T}\left\|\int_{0}^{t} Z_{\alpha, \alpha}(t-s) A f(s) d s\right\|_{\mathfrak{Z}}^{q} d t \leqslant \\
& \leqslant C \int_{0}^{T}\left(\int_{0}^{t}\|A f(s)\|_{\mathfrak{Z}} d s\right)^{q} d t \leqslant C \int_{0}^{T} t^{q-1} d t\|f\|_{L_{q}\left(0, T ; D_{A}\right)}^{q}=\frac{C T^{q}}{q}\|f\|_{L_{q}\left(0, T ; D_{A}\right)}^{q} .
\end{aligned}
$$

При $\alpha \in(0,1)$ согласно (6) получим

$$
\begin{array}{r}
\left\|A Z_{f}\right\|_{L_{q}(0, T ; \mathfrak{Z})}^{q} \leqslant \int_{0}^{T}\left\|\int_{0}^{t} Z_{\alpha, \alpha}(t-s) A f(s) d s\right\|_{\mathfrak{Z}}^{q} d t \leqslant C_{1} \int_{0}^{T}\left(\int_{0}^{t}(t-s)^{\alpha-m}\|A f(s)\|_{\mathfrak{Z}} d s\right)^{q} d t \leqslant \\
\leqslant C_{2} \int_{0}^{T} t^{(\alpha-m+1) q-1} d t\|f\|_{L_{q}\left(0, T ; D_{A}\right)}^{q}=\frac{C_{2} T^{(\alpha-m+1) q}}{(\alpha-m+1) q}\|f\|_{L_{q}\left(0, T ; D_{A}\right)}^{q} .
\end{array}
$$

Аналогично доказывается, что $Z_{f} \in L_{q}(0, T ; \mathfrak{Z})$, поэтому $Z_{f}$ - искомое сильное решение.

Если существуют решения $\hat{z}_{1}$ и $\hat{z}_{2}$ задачи (8), (9), то их разность $z=\hat{z}_{1}-\hat{z}_{2}$ является решением задачи Коши (8) с $z_{k}=0, k=0,1, \ldots, m-1$, для уравнения (5). Используя преобразование Лапласа для однородного уравнения, получим

$$
\mathfrak{L}\left[D_{t}^{\alpha} z\right](\lambda)=\lambda^{\alpha} \mathfrak{L}[z](\lambda)=A \mathfrak{L}[z](\lambda) ;
$$

следовательно, $\mathfrak{L}[z](\lambda) \equiv 0$ в полуплоскости $\left\{\lambda \in \mathbb{C}: \operatorname{Re} \lambda>a_{0}\right\}$, поскольку $A \in \mathcal{A}^{\alpha}\left(\theta_{0}, a_{0}\right)$. Таким образом, $z(t) \equiv 0$.

Оценка (10) почти очевидна; заметим лишь, что при ее получении используются равенства

$$
\begin{aligned}
D_{t}^{\alpha}\left(\sum_{k=0}^{m-1} Z_{\alpha, k+1}(t) z_{k}+\int_{0}^{t} Z_{\alpha, \alpha}(t-s) f(s) d s\right) & =A \sum_{k=0}^{m-1} Z_{\alpha, k+1}(t) z_{k}+A \int_{0}^{t} Z_{\alpha, \alpha}(t-s) f(s) d s+f(t)= \\
& =\sum_{k=0}^{m-1} Z_{\alpha, k+1}(t) A z_{k}+\int_{0}^{t} Z_{\alpha, \alpha}(t-s) A f(s) d s+f(t) .
\end{aligned}
$$


3. Разрешимость задач оптимального управления для линейного уравнения дробного порядка с секториальным оператором. Пусть $\mathfrak{Z}, \mathcal{U}$-банаховы пространства, $A \in$ $\mathcal{A}^{\alpha}\left(\theta_{0}, a_{0}\right), B \in \mathcal{L}(\mathcal{U} ; \mathfrak{Z}), f \in(0, T) \rightarrow \mathfrak{Z}$. Рассмотрим задачу управления

$$
\begin{gathered}
D_{t}^{\alpha} z(t)=A z(t)+B u(t)+f(t), \quad t \in(0, T), \\
z^{(k)}(0)=z_{k}, \quad k=0,1, \ldots, m-1, \\
u \in \mathcal{U}_{\partial} \\
J(z, u) \rightarrow \inf
\end{gathered}
$$

где $\mathcal{U}_{\partial}$ - множество допустимых управлений, $J$ - функционал качества, $m-1<\alpha \leqslant m \in \mathbb{N}$. Введем в рассмотрение пространство

$$
\mathcal{Q}_{\alpha, q}(0, T ; \mathfrak{Z}) \equiv\left\{z \in C^{m-1}([0, T] ; \mathfrak{Z}): J_{t}^{m-\alpha}\left(z-\sum_{k=0}^{m-1} z^{(k)}(0) g_{k+1}\right) \in W_{q}^{m}(0, T ; \mathfrak{Z})\right\} .
$$

Лемма 2. Пространство $\mathcal{Q}_{\alpha, q}(0, T ; \mathfrak{Z})$ является банаховым пространством с нормой

$$
\|z\|_{\mathcal{Q}_{\alpha, q}(0, T ; \mathfrak{3})}=\|z\|_{C^{m-1}([0, T] ; \mathfrak{Z})}+\left\|D_{t}^{\alpha} z\right\|_{L_{q}(0, T ; \mathfrak{3})} \cdot
$$

Доказательство. По сути необходимо доказать замкнутость оператора

$$
D_{t}^{\alpha}: C^{m-1}([0, T] ; \mathfrak{Z}) \rightarrow L_{q}(0, T ; \mathfrak{Z})
$$

с областью определения $\mathcal{Q}_{\alpha, q}(0, T ; \mathfrak{Z})$. Представим его в виде $D_{t}^{\alpha}={ }^{R} D_{t}^{\alpha} S_{m}$, где ${ }^{R} D_{t}^{\alpha}=D_{t}^{m} J_{t}^{m-\alpha}-$ дробная производная Римана-Лиувилля,

$$
S_{m} z \equiv z-\sum_{k=0}^{m-1} z^{(k)}(0) g_{k+1} .
$$

Очевидно, что оператор $S_{m}$ действует непрерывно из $\mathcal{Q}_{\alpha, q}(0, T ; \mathfrak{Z})$ с нормой пространства $C^{m-1}([0, T] ; \mathfrak{Z})$ в пространство

$$
\mathcal{R}_{\alpha, q, 0} \equiv\left\{z \in L_{q}(0, T ; \mathfrak{Z}): J_{t}^{m-\alpha} z \in W_{q, 0}^{m}(0, T ; \mathfrak{Z})\right\},
$$

снабженное нормой $L_{q}(0, T ; \mathfrak{Z})$. Оператор же ${ }^{R} D_{t}^{\alpha}: \mathcal{R}_{\alpha, q, 0} \rightarrow L_{q}(0, T ; \mathfrak{Z})$ замкнут в силу леммы $1.8(\mathrm{a})$ из $[10$, с. 15].

Лемма 3. При $q(m-\alpha)>1$ пространство $W_{q}^{m}(0, T ; \mathfrak{Z})$ непрерывно вложено в $\mathcal{Q}_{\alpha, q}(0, T ; \mathfrak{Z})$.

Доказательство. Пространство $W_{q}^{m}(0, T ; \mathfrak{Z})$ непрерывно вложено в $C^{m-1}([0, T] ; \mathfrak{Z})$. Используя этот факт, а также неравенство Гельдера, получим:

$$
\begin{gathered}
\|z\|_{\mathcal{Q}_{\alpha, q}(0, T ; \mathfrak{3})} \leqslant C_{1}\|z\|_{W_{q}^{m}(0, T ; \mathfrak{3})}+\left\|D_{t}^{m} \int_{0}^{t} \frac{(t-s)^{m-\alpha-1}}{\Gamma(m-\alpha)}\left(z(s)-\sum_{k=0}^{m-1} z^{(k)}(0) \frac{(s)^{k}}{k !}\right) d s\right\|_{L_{q}(0, T ; \mathfrak{3})}= \\
=C_{1}\|z\|_{W_{q}^{m}(0, T ; \mathfrak{3})}+\left\|D_{t}^{m} \int_{0}^{t} \frac{\tau^{m-\alpha-1}}{\Gamma(m-\alpha)}\left(z(t-\tau)-\sum_{k=0}^{m-1} z^{(k)}(0) \frac{(t-\tau)^{k}}{k !}\right) d \tau\right\|_{L_{q}(0, T ; \mathfrak{3})}= \\
=C_{1}\|z\|_{W_{q}^{m}(0, T ; \mathfrak{3})}+\left\|\int_{0}^{t} \frac{\tau^{m-\alpha-1}}{\Gamma(m-\alpha)} z^{(m)}(t-\tau) d \tau\right\|_{L_{q}(0, T ; \mathfrak{3})} \leqslant
\end{gathered}
$$




$$
\begin{aligned}
& \leqslant C_{1}\|z\|_{W_{q}^{m}(0, T ; \mathfrak{Z})}+\left(\int_{0}^{T}\left(\int_{0}^{t} \frac{s^{\frac{(m-\alpha-1) q}{q-1}}}{\Gamma(m-\alpha)} d s\right)^{q-1} \int_{0}^{t}\left\|z^{(m)}(s)\right\|_{\mathfrak{Z}}^{q} d s d t\right)^{1 / q} \leqslant \\
& \leqslant\left(C_{1}+\left(\frac{q-1}{\Gamma(m-\alpha)((m-\alpha) q-1)}\right)^{1-1 / q} \frac{T^{m-\alpha}}{((m-\alpha) q)^{1 / q}}\right)\|z\|_{W_{q}^{m}(0, T ; \mathfrak{3})}
\end{aligned}
$$

Решение $z$ задачи (11), (12) по определению ищется в пространстве $\mathcal{Z}_{\alpha, q}=\mathcal{Q}_{\alpha, q}(0, T ; \mathfrak{Z}) \cap$ $L_{q}\left(0, T ; D_{A}\right)$. Как пересечение двух банаховых пространств, $\mathcal{Z}_{\alpha, q}$ также является банаховым пространством, наделенным нормой

$$
\|z\|_{\mathcal{Z}_{\alpha, q}}=\|z\|_{\mathcal{Q}_{\alpha, q}(0, T ; \mathfrak{3})}+\|A z\|_{L_{q}(0, T ; \mathfrak{Z})}
$$

Введем в рассмотрение оператор $\gamma_{0} z=z(0), \gamma_{0}: C([0, T] ; \mathfrak{Z}) \rightarrow \mathfrak{Z}$. Естественно, он является непрерывным на пространстве $\mathcal{Q}_{\alpha, q}(0, T ; \mathfrak{Z})$ и на $\mathcal{Z}_{\alpha, q}$, т.е. $\gamma_{0} \in \mathcal{L}\left(\mathcal{Z}_{\alpha, q} ; \mathfrak{Z}\right)$.

Множество пар $(z, u)$ будем называть множеством допустимых пар $\mathfrak{W}$ задачи (11)-(14), если $z \in \mathcal{Z}_{\alpha, q}$ - сильное решение задачи (11), (12) при условии, что $u \in \mathcal{U}_{\partial}$ и $J(z, u)<\infty$.

Задача (11)-(14) заключается в нахождении пар $(\hat{z}, \hat{u}) \in \mathfrak{W}$, минимизирующих функционал качества: $J(\hat{z}, \hat{u})=\inf _{(z, u) \in \mathfrak{W}} J(z, u)$.

Теорема 2. Пусть $\alpha>0, q>(\alpha-m+1)^{-1}, f \in L_{q}\left(0, T ; D_{A}\right), \mathcal{U}_{\partial}-$ непустое выпуклое замкнутое подмножество пространства $L_{q}(0, T ; \mathcal{U}), \mathcal{U}_{\partial} \cap L_{q}\left(0, T ; D_{A}\right) \neq \emptyset$, в банахово пространство $\mathfrak{Y} \subset L_{q}(0, T ; \mathfrak{Z})$ непрерывно вложено $\mathcal{Z}_{\alpha, q}, J$-выпуклый функиионал качества, ограниченный снизу и полунепрерывный снизу на $\mathfrak{Y} \times L_{q}(0, T ; \mathcal{U})$, коэрцитивный на пространстве $\mathcal{Z}_{\alpha, q} \times L_{q}(0, T ; \mathcal{U}), z_{k} \in D_{A}, k=0,1, \ldots, m-1$. Тогда существует решение $(\hat{z}, \hat{u}) \in \mathcal{Z}_{\alpha, q} \times \mathcal{U}_{\partial}$ задачи (11)-(14). Если функиионал J является строго выпуклым на $\mathfrak{Y} \times L_{q}(0, T ; \mathcal{U})$, то решение задачи (11)-(14) единственно.

Доказательство. По теореме 1 при любом $u \in \mathcal{U}_{\partial} \cap L_{q}\left(0, T ; D_{A}\right)$ существует единственное сильное решение задачи (11), (12). Поэтому множество допустимых пар $\mathfrak{W}$ непусто. Положим

$$
\mathfrak{Y}_{1}=\mathcal{Z}_{\alpha, q}, \quad \mathfrak{U}=L_{q}(0, T ; \mathcal{U}), \quad \mathfrak{V}=L_{q}(0, T ; \mathfrak{Z}) \times \mathfrak{Z}^{m}, \quad \mathfrak{F}=-\left(f, z_{0}, z_{1}, \ldots, z_{m-1}\right) \in \mathfrak{V},
$$

линейный оператор

$$
\mathfrak{L}(z, u)=\left(D_{t}^{\alpha} z-A z-B u, \gamma_{0} z, \gamma_{0} z^{(1)}, \ldots, \gamma_{0} z^{(m-1)}\right) .
$$

Тогда система управления (11), (12) представлена в виде $\mathfrak{L}(z, u)+\mathfrak{F}=0$.

Непрерывность оператора $\mathfrak{L}: \mathfrak{Y}_{1} \times \mathfrak{U} \rightarrow \mathfrak{V}$ следует из неравенств

$$
\begin{aligned}
\left\|\left(D_{t}^{\alpha} z-A z-B u, \gamma_{0} z, \gamma_{0} z^{(1)}, \ldots, \gamma_{0} z^{(m-1)}\right)\right\|_{L_{q}(0, T ; \mathfrak{Z}) \times \mathfrak{Z}^{m}} & \\
\leqslant C_{1}\left(\|z\|_{\mathcal{Q}_{\alpha, q}(0, T ; \mathfrak{3})}+\|z\|_{L_{q}\left(0, T ; D_{A}\right)}+\|u\|_{L_{q}(0, T ; \mathcal{U})}+\right. & \left.\|z\|_{C^{m-1}([0, T] ; \mathfrak{3})}\right) \leqslant \\
& \leqslant C_{2}\left(\|z\|_{\mathcal{Z}_{\alpha, q}}+\|u\|_{L_{q}(0, T ; \mathcal{U})}\right) .
\end{aligned}
$$

По теореме 2.3 из [9] получим требуемое.

Замечание 1. Выбор пространства $\mathfrak{Y}$ при применении теоремы 2 определяется видом функционала $J$. Проиллюстрируем это на следующих примерах.

Рассмотрим задачу минимизации функционала:

$$
J(z, u)=\left\|z-z_{r}\right\|_{\mathcal{Q}_{\alpha, q}(0, T ; \mathfrak{Z})}^{q}+\delta\left\|u-u_{r}\right\|_{L_{q}(0, T ; \mathcal{U})}^{q} \rightarrow \inf
$$

при заданных $z_{r} \in \mathcal{Q}_{\alpha, q}(0, T ; \mathfrak{Z}), u_{r} \in L_{q}(0, T ; \mathcal{U}), \delta>0$.

Теорема 3. Пусть $\alpha>0, q>(\alpha-m+1)^{-1}, f \in L_{q}\left(0, T ; D_{A}\right), \mathcal{U}_{\partial}-$ непустое выпуклое замкнутое подмножество в $L_{q}(0, T ; \mathcal{U}), \mathcal{U}_{\partial} \cap L_{q}\left(0, T ; D_{A}\right) \neq \emptyset, z_{k} \in D_{A}, k=0,1, \ldots, m-1$. Тогда существует решение $(\hat{z}, \hat{u}) \in \mathcal{Z}_{\alpha, q} \times \mathcal{U}_{\partial}$ задачи (11)-(13), (15). 
Доказательство. Достаточно взять $\mathfrak{Y}=\mathcal{Q}_{\alpha, q}(0, T ; \mathfrak{Z})$, чтобы все условия теоремы 2 выполнялись. Действительно, выпуклость, ограниченность снизу и непрерывность на $\mathfrak{Y} \times \mathfrak{U}$ функционала $J$ очевидны; при этом

$$
\begin{gathered}
\|z\|_{\mathcal{Z}_{\alpha, q}}+\|u\|_{L_{q}(0, T ; \mathcal{U})}=\|z\|_{\mathcal{Q}_{\alpha, q}(0, T ; \mathfrak{3})}+\|z\|_{L_{q}(0, T ; \mathfrak{Z})}+\|A z\|_{L_{q}(0, T ; \mathfrak{3})}+\|u\|_{L_{q}(0, T ; \mathcal{U})}= \\
=\|z\|_{\mathcal{Q}_{\alpha, q}(0, T ; \mathfrak{3})}+\|z\|_{L_{q}(0, T ; \mathfrak{3})}+\left\|D_{t}^{\alpha} z-B u-f\right\|_{L_{q}(0, T ; \mathfrak{3})}+\|u\|_{L_{q}(0, T ; \mathcal{U})} \leqslant \\
\leqslant C_{1}\left(\|z\|_{\mathcal{Q}_{\alpha, q}(0, T ; \mathfrak{3})}+\|u\|_{L_{q}(0, T ; \mathcal{U})}+\|f\|_{L_{q}(0, T ; \mathfrak{3})}\right) \leqslant C_{1}+C_{2}\left(J(z, u)+C_{3}\right)^{1 / q} \leqslant C_{1}+C_{2}\left(R+C_{3}\right)^{1 / q}
\end{gathered}
$$

если $J(z, u) \leqslant R$, что и означает коэрцитивность функционала на $\mathfrak{Y}_{1} \times \mathfrak{U}$.

Для функционала

$$
J_{q}(z, u)=\left\|z-z_{r}\right\|_{W_{q}^{l}(0, T ; \mathfrak{3})}^{q}+\delta\left\|u-u_{r}\right\|_{L_{q}(0, T ; \mathcal{U})}^{q} \rightarrow \inf
$$

при $l \in\{0,1, \ldots, m-1\}, z_{r} \in W_{q}^{l}(0, T ; \mathfrak{Z}), u_{r} \in L_{q}(0, T ; \mathcal{U}), \delta>0$ докажем следующее утверждение.

Теорема 4. Пусть $\alpha>0, q>(\alpha-m+1)^{-1}, f \in L_{q}\left(0, T ; D_{A}\right), \mathcal{U}_{\partial}$ - непустое выпуклое замкнутое подмножество пространства $L_{q}(0, T ; \mathcal{U}), \mathcal{U}_{\partial} \cap L_{q}\left(0, T ; D_{A}\right) \neq \emptyset, z_{k} \in D_{A}, k=0,1, \ldots, m-1$. Тогда существует решение $(\hat{z}, \hat{u}) \in \mathcal{Z}_{\alpha, q} \times \mathcal{U}_{\partial}$ задачи (11)-(13), (16). Если $\mathfrak{Z}, \mathcal{U}$ - гильбертовы пространства, а $q=2$, то решение задачи (11)-(13), (16) единственно.

Доказательство. Пространство $\mathfrak{Y}=W_{q}^{l}(0, T ; \mathfrak{Z})$ непрерывно вложено в $\mathcal{Z}_{\alpha, q}$. Используя оценку (10) для сильного решения, получим по аналогии с предыдущим доказательством:

$$
\begin{aligned}
& \|z\|_{\mathcal{Z}_{\alpha, q}}+\|u\|_{L_{q}(0, T ; \mathcal{U})} \leqslant C_{1}\left(\|z\|_{\mathcal{Q}_{\alpha, q}(0, T ; \mathfrak{Z})}+\|u\|_{L_{q}(0, T ; \mathcal{U})}+\|f\|_{L_{q}(0, T ; \mathfrak{Z})}\right) \leqslant \\
& \leqslant C_{2}\left(\|u\|_{L_{q}(0, T ; \mathcal{U})}+\sum_{k=0}^{m-1}\left\|z_{k}\right\|_{D_{A}}+\|f\|_{L_{q}\left(0, T ; D_{A}\right)}\right) \leqslant C_{3}\left(J(z, u)+C_{4}\right)^{1 / q}+C_{5} .
\end{aligned}
$$

Отсюда следует коэрцитивность функционала на $\mathfrak{Y}_{1} \times \mathfrak{U}$.

Если $\mathfrak{Z}, \mathcal{U}$ - гильбертовы пространства, $q=2$, то функционал $J_{2}$ из $(16)$ является строго выпуклым как квадрат нормы в гильбертовом пространстве $W_{2}^{l}(0, T ; \mathfrak{Z}) \times L_{2}(0, T ; \mathcal{U})$. По теореме 2.3 из [9] получим единственность решения задачи (11)-(13), (16).

4. Задачи оптимального управления для уравнения субдиффузии и диффузионно-волнового уравнения. Приведем примеры задач оптимального управления для уравнения дробного порядка $\alpha \in(0,2)$ по времени, представляющего собой уравнение субдиффузии при $\alpha \in(0,1)$ и диффузионно-волновое уравнение при $\alpha \in(1,2)$.

Пусть $\Omega$ - ограниченная область в $\mathbb{R}^{d}$ с гладкой границей $\partial \Omega$. Рассмотрим систему управления, описываемую начально-краевой задачей

$$
\begin{gathered}
\frac{\partial^{k} v}{\partial t^{k}}(x, 0)=v_{k}(x), \quad k=0,1, \ldots, m-1, \quad x \in \Omega, \\
v(x, t)=0, \quad(x, t) \in \partial \Omega \times(0, T), \\
D_{t}^{\alpha} v(x, t)=\nu \Delta v(x, t)+u(x, t), \quad(x, t) \in \Omega \times(0, T) .
\end{gathered}
$$

Выберем пространство $\mathfrak{Z}=L_{2}(\Omega)$ и оператор

$$
A=\Delta=\frac{\partial^{2}}{\partial x_{1}^{2}}+\frac{\partial^{2}}{\partial x_{2}^{2}}+\cdots+\frac{\partial^{2}}{\partial x_{d}^{2}}, \quad D_{A}=H_{0}^{2}(\Omega) \equiv\left\{w \in H^{2}(\Omega): w(x)=0, x \in \partial \Omega\right\} .
$$

Тогда

$$
\mathcal{Z}_{\alpha, q}=\mathcal{Q}_{\alpha, q}\left(0, T ; L_{2}(\Omega)\right) \cap L_{q}\left(0, T ; H_{0}^{2}(\Omega)\right) .
$$

Возьмем $\mathcal{U}=L_{2}(\Omega)$ и рассмотрим задачу управления

$$
\begin{gathered}
u \in \mathcal{U}_{\partial} \equiv\left\{u \in L_{q}\left(0, T ; L_{2}(\Omega)\right):\|u\|_{L_{q}\left(0, T ; L_{2}(\Omega)\right)} \leqslant R\right\}, \\
J(v, u)=\left\|v-v_{r}\right\|_{W_{q}^{l}\left(0, T ; L_{2}(\Omega)\right)}^{q}+\delta\left\|u-u_{r}\right\|_{L_{q}\left(0, T ; L_{2}(\Omega)\right)}^{q} \rightarrow \inf
\end{gathered}
$$

при заданных $l \in\{0,1, \ldots, m-1\}, v_{r} \in W_{q}^{l}\left(0, T ; H_{0}^{2}(\Omega)\right), u_{r} \in L_{q}\left(0, T ; L_{2}(\Omega)\right), R>0, \delta>0$. 
Лемма 4 (см. [8]). Пусть $\alpha>1, A \in \mathcal{C l}(\mathfrak{Z})$,

$$
\begin{aligned}
& \exists \theta_{1} \in(\pi / 2, \pi) \exists a_{1} \geqslant 0 \forall \mu \in S_{a_{1}, \theta_{1}} \mu^{\alpha} \in \rho^{L}(M) ; \\
& \exists C_{1}>0 \forall \mu \in S_{a_{1}, \theta_{1}}\left\|R_{\mu^{\alpha}}(A)\right\|_{\mathcal{L}(\mathfrak{Z})} \leqslant \frac{C_{1}}{\left|\mu^{\alpha}-a_{1}\right|} .
\end{aligned}
$$

Тогда $A \in \mathcal{A}^{\alpha}\left(\theta_{0}, a_{0}\right)$ при некоторых $\theta_{0} \in\left(\pi / 2, \theta_{1}\right), a_{0} \geqslant a_{1}, a_{0}>1$.

Лемма 5 (см. [8]). Пусть $\alpha \in(0,1), A \in \mathcal{C l}(\mathfrak{Z})$,

$$
\begin{gathered}
\exists \theta_{1} \in(\pi / 2, \pi) \exists a_{0} \in[0,1) \forall \mu \in S_{a_{0}, \theta_{1}} \mu^{\alpha} \in \rho^{L}(M) ; \\
\exists C_{1}>0 \forall \mu \in S_{a_{0}, \theta_{1}}\left\|R_{\mu^{\alpha}}(A)\right\|_{\mathcal{L}(\mathfrak{Z})} \leqslant \frac{C_{1}}{\left|\mu^{\alpha}-a_{0}\right|} .
\end{gathered}
$$

Тогда $A \in \mathcal{A}^{\alpha}\left(\theta_{0}, a_{0}\right)$ для некоторого $\theta_{0} \in\left(\pi / 2, \theta_{1}\right)$.

Замечание 2. Леммы 4 и 5 были доказаны в [8] в других терминах, но после замены выражения

$$
\max \left\{\left\|R_{\mu^{\alpha}}^{L}(M)\right\|_{\mathcal{L}(\mathfrak{X})},\left\|L_{\mu^{\alpha}}^{L}(M)\right\|_{\mathcal{L}(\mathfrak{Y})}\right\}
$$

в условиях лемм на $\left\|R_{\mu^{\alpha}}(A)\right\|_{\mathcal{L}(\mathfrak{3})}$ получатся сформулированные здесь утверждения.

Теорема 5. Пусть $\nu>0, \alpha \in(0,2), q>(\alpha-m+1)^{-1}, v_{k} \in H_{0}^{2}(\Omega), k=0,1, \ldots, m-1$, $\mathcal{U}_{\partial} \cap L_{q}\left(0, T ; H_{0}^{2}(\Omega)\right) \neq \emptyset$. Тогда существует решение $(\hat{v}, \hat{u}) \in \mathcal{Z}_{\alpha, q} \times \mathcal{U}_{\partial}$ задачи $(17)-(21)$. Если $q=2$, то решение единственно.

Доказательство. Спектр $\sigma(A)$ оператора $A$ вещественный, дискретный, конечнократный и сгущается к $-\infty$. Пусть $\left\{\varphi_{k}: k \in \mathbb{N}\right\}$ - ортонормированная в $L_{2}(\Omega)$ система собственных функций оператора $A$, занумерованных по невозрастанию соответствующих собственных значений $\left\{\lambda_{k}: k \in \mathbb{N}\right\}$ с учетом их кратности. Для $w \in L_{2}(\Omega)$ имеем

$$
R_{\mu^{\alpha}}(A) w=\sum_{k=1}^{\infty} \frac{\left\langle w, \varphi_{k}\right\rangle \varphi_{k}}{\mu^{\alpha}-\lambda_{k}}, \quad\left\|R_{\mu^{\alpha}}(A) w\right\|_{L_{2}(\Omega)}^{2}=\sum_{k=1}^{\infty} \frac{\left|\left\langle w, \varphi_{k}\right\rangle\right|^{2}}{\left|\mu^{\alpha}-\lambda_{k}\right|^{2}} \leqslant \frac{\sin ^{-2} \theta_{1}}{|\mu|} \sum_{k=1}^{\infty}\left|\left\langle w, \varphi_{k}\right\rangle\right|^{2}
$$

при любом $\theta_{1} \in(\pi / 2, \pi)$. Здесь $\langle\cdot, \cdot\rangle$ - скалярное произведение в $L_{2}(\Omega)$.

При $\alpha>1$ возьмем любое $\theta_{1} \in(\pi / 2, \pi / \alpha)$; тогда для $\mu \in S_{0, \theta_{1}}$ будет выполняться условие $\mu^{\alpha} \in(\pi / 2, \pi)$, поэтому $\mu^{\alpha} \in \rho(A)$ в силу вещественности спектра $\sigma(A)$. По лемме 4 получим, что $A \in \mathcal{A}^{\alpha}\left(\theta_{0}, a_{0}\right)$ при некоторых $a_{0}>1, \theta_{0} \in(\pi / 2, \pi / \alpha)$. При $\alpha<1$ с помощью леммы 5 аналогично доказываем, что $A \in \mathcal{A}^{\alpha}\left(\theta_{0}, a_{0}\right)$ при $a_{0}=0, \theta_{0} \in(\pi / 2, \pi / \alpha)$. При $\alpha=1$ доказываемое утверждение в точности означает известный факт, что данный оператор $A$ порождает аналитическую в секторе $\left(C_{0}\right)$-полугруппу.

Таким образом, начально-краевая задача (17)-(19), редуцированная к задаче (8), (9), по теореме 1 имеет единственное сильное решение при каждом $u \in \mathcal{U}_{\partial} \cap L_{q}\left(0, T ; H_{0}^{2}(\Omega)\right)$. Поэтому выполняется условие нетривиальности для задачи оптимального управления (11)-(13), (16), к которой сведена исследуемая задача (17)-(21).

Множество допустимых управлений, заданное в (20), является выпуклым замкнутым подмножеством в пространстве $L_{q}(0, T ; \mathcal{U})$. Опираясь на теорему 4 , получим существование оптимального управления. Его единственность при $q=2$ следует из той же теоремы с учетом гильбертовости пространства $\mathfrak{Z}=\mathcal{U}=L_{2}(\Omega)$.

Рассмотрим функционал

$$
J(z, u)=\left\|v-v_{r}\right\|_{\mathcal{Q}_{\alpha, q}\left(0, T ; L_{2}(\Omega)\right)}^{q}+\delta\left\|u-u_{r}\right\|_{L_{q}\left(0, T ; L_{2}(\Omega)\right)}^{q} \rightarrow \inf
$$

при заданных $v_{r} \in \mathcal{Q}_{\alpha, q}\left(0, T ; L_{2}(\Omega)\right), u_{r} \in L_{q}\left(0, T ; L_{2}(\Omega)\right), \delta>0$. Рассуждая, как при рассмотрении предыдущей задачи, но используя теорему 3 , нетрудно доказать следующее утверждение.

Теорема 6. Пусть $\nu>0, \alpha \in(0,2), q>(\alpha-m+1)^{-1}, v_{k} \in H_{0}^{2}(\Omega), k=0,1, \ldots, m-1$, $\mathcal{U}_{\partial} \cap L_{q}\left(0, T ; H_{0}^{2}(\Omega)\right) \neq \emptyset$. Тогда существует решение $(\hat{v}, \hat{u}) \in \mathcal{Z}_{\alpha, q} \times \mathcal{U}_{\partial}$ задачи (17)-(20), (22). 


\section{СПИСОК ЛИТЕРАТУРЫ}

1. Иосида К. Функциональный анализ. - М.: Мир, 1967.

2. Клемент Ф., Хейманс Х., Ангенент С., ван Дуйн К., де Пахтер Б. Однопараметрические полугруппы. - М.: Мир, 1992.

3. Костин В. А. К теореме Соломяка-Иосиды для аналитических полугрупп// Алгебра и анализ. 1999. - 11, № 1. - C. 118-140.

4. Плеханова М. В. Задачи оптимального управления для линейных вырожденных дробных уравнений// Итоги науки и техн. Совр. мат. прилож. Тематич. обзоры. - 2018. - 149. - С. $72-83$.

5. Соломлк М. 3. Применение теории полугрупп к исследованию дифференциальных уравнений в пространствах Банаха// Докл. АН СССР. - 1958. - 122, № 5. - С. 766-769.

6. Романова E. A., Федоров B. E. Разрешающие операторы линейного вырожденного эволюционного уравнения с производной Капуто. Секториальный случай// Мат. заметки СВФУ. - 2016. - 23, № 4 (92). - C. $58-72$.

7. Федоров В. Е., Гордиевских Д. М., Плеханова М. В. Уравнения в банаховых пространствах с вырожденным оператором под знаком дробной производной// Диффер. уравн. - 2015. - 51, № 10. C. $1367-1375$.

8. Федоров В. Е., Романова Е. А., Дебуш А. Аналитические в секторе разрешающие семейства операторов вырожденных эволюционных уравнений дробного порядка// Сиб. ж. чист. прикл. мат. - 2016. 16, № 2. - C. 93-107.

9. Фурсиков А. В. Оптимальное управление распределенными системами. Теория и приложения. - Новосибирск: Научная книга, 1999.

10. Bajlekova E. G. Fractional Evolution Equations in Banach Spaces/ PhD thesis - Eindhoven: Eindhoven Univ. of Technology, 2001.

11. Plekhanova $M$. V. Strong solutions to nonlinear degenerate fractional order evolution equations// J. Math. Sci. - 2018. - 230, № 1. - P. 146-158.

12. Prüss J. Evolutionary Integral Equations and Applications. — Basel: Springer, 1993.

Плеханова Марина Васильевна

Южно-Уральский государственный университет

(национальный исследовательский университет), Челябинск;

Челябинский государственный университет

E-mail: mariner79@mail.ru 